Math. Z. 128, 297-304 (1972)

(C) by Springer-Verlag 1972

\title{
Groups with a Steinberg Character
}

\author{
Forrest A. Richen
}

Let $G$ be a finite group and $p$ a rational prime. If $G$ is a simple Lie type group of characteristic $p$ then $G$ has the following property. $G$ has an absolutely irreducible character $\chi$ whose degree is the order of a $p$-Sylow subgroup of $G$, and all other characters are in the principal $p$-block, $B_{0}(p)$. ( $\chi$ is called the Steinberg character. See [4, 5, 9] and [12].) For simple groups $G$, this property seems to hold only if $G$ has Lie type of characteristic $p$.

The most general conjecture that can be made from this observation, namely that the above property about the characters of $G$ forces $G$ to be of Lie type at characteristic $p$, seems too difficult at present. In fact to prove anything in this direction, I have had to make arithemtical assumptions about $G$ as well as assumptions about a subgroup which ultimately corresponds to the normalizer of a torus. In Section 4 I will indicate why such assumptions may be inevitable.

Theorem. Let $G$ be a finite group and $p$ a prime such that $|G|=p g^{\prime}$, $\left(p, g^{\prime}\right)=1$. Suppose that $G$ has an irreducible character $\chi$ of degree $p$ and that all other characters of $G$ are in the principal p-block, $B_{0}(p)$. Let $P$ be a p-Sylow subgroup and let $N(P)=H P$ where $H \cap P=\{1\}$. Suppose that $H$ is a TI set and $|N(H)|=2|H|$. Then $G$ is isomorphic to $P S L_{2}(p)$.

(Recall a subgroup $A$ of a group $G$ is a $T I$ set if $A \cap A^{g} \neq\{1\}$ implies $g \in N(A)$.)

The following corollary is related to a theorem of Ito [11].

Corollary. Let $G$ be a transitive permutation group of prime degree, p. Suppose $G$ has a unique character $\chi$ whose degree is divisible by $p$ and that $\chi(1)=p$. Suppose that if the normalizer of a $p$-Sylow subgroup $P$ of $G$ is $H P$ where $H \cap P=\{1\}$ then $H$ is $a T I$ set and $|N(H)|=2|H|$. Then $p=5,7$ or 11 and $G \cong P S L_{2}(p)$.

The proof of the theorem is carried out in Sections 1-3 and the corollary is proved and discussed in Section 4 . I assume familiarity with elementary finite group theory and character theory including the theory of blocks. (Gorenstein's book [8], Huppert's book [10] and Curtis and Reiner's book [3] will serve as general references.) At a crucial point detailed information about $B_{0}(p)$ is needed for groups containing $p$ to the first power (Brauer [1]), but that information is fully stated in the text. 


\section{$\S 1$. Some Subgroups of $G$}

Let $G$ be a group that satisfies the hypotheses of the theorem. Let $P$ be a $p$-Sylow subgroup, $B=N(P)=H P$ where $H \cap P=1$, and $N=N(H)$. Since the characters of $G$ are $\{\chi\} \cup B_{0}(p), G$ has exactly one block of defect 1. Brauer's first main theorem gives $C(P) \subseteq P$ (or see Theorem 3 in Brauer [2]). Thus $H \cong N(P) / C(P)$ is a cyclic group whose order $e$ divides $p-1$. Since $|N|=2 e, e=1$ would give $|G|=2$ and $p=2$ contrary to the assumption that $G$ has a character of degree 2 . Thus $e>1$. This together with Burnside's fusion theorem (7.1.1, [8]) gives the following fact.

(1.1) $B$ is a Frobenius group of order pe with cyclic complement $H$ of order $e>1$. $G$ has $t=(p-1) / e$ classes of $p$-elements.

(1.2) $G$ is a non abelian simple group.

Proof. Suppose $1 \neq S \triangle G$ and $p|| G: S \mid$. Then $S \leqq O_{p^{\prime}}(G)$ which is just $\cap\left\{\operatorname{ker} \zeta: \zeta \in B_{0}(p)\right\}$. (Theorem 1, Brauer [2].) Thus the characters of $G / O_{p^{\prime}}(G)$ are just the characters of $B_{0}(p)$. Then summing the squares of the degrees of the characters of $G$ gives

$$
|G|=\sum_{\zeta \in B_{0}(p)} \zeta(1)^{2}+p^{2}=\left|G: O_{p^{\prime}}(G)\right|+p^{2} .
$$

The only solution to this equation is $|G|=(p+1) p$. But $e \mid(p-1)$ and $e \mid(p+1) p$ forces $e=1$ against (1.1).

On the other hand suppose $p|| S \mid$ and $S \triangle G$. Then $P \leqq S$, and the Frattini Lemma (1.3.7, [8]) gives $G=S B$. Hence $G / S \cong B / S \cap B$ is cyclic of order prime to $p$. Thus $G^{\prime} \subseteq S$ and we may assume $p|| G^{\prime} \mid$. Now $\chi \varphi$ is an irreducible character of degree $p$ if $\varphi$ is a linear character of $G$. Since $\chi$ is the unique irreducible character of degree $p, \chi \varphi=\chi$. Thus $\chi$ vanishes on $G-G^{\prime}$. Hence

$$
\begin{aligned}
\left(\left.\chi\right|_{G^{\prime}},\left.\chi\right|_{G^{\prime}}\right) & =\frac{1}{\left|G^{\prime}\right|} \sum_{x \in G^{\prime}}|\chi(x)|^{2} \\
& =\frac{1}{\left|G^{\prime}\right|} \sum_{x \in G}|\chi(x)|^{2} \\
& =\left|G: G^{\prime}\right| .
\end{aligned}
$$

Now Clifford's Theorem (V, 17.3 in [10]) gives

$$
\left.\chi\right|_{G^{\prime}}=e \sum_{i=1}^{n} \lambda_{i}
$$

where $e$ is some positive integer and the $\lambda_{i}$ 's are the distinct $G$-conjugates of some irreducible character $\lambda_{1}$ of $G^{\prime}$. Taking degrees shows $p=e n \lambda_{1}(1)$. 
Taking the inner product of $\left.\chi\right|_{G^{\prime}}$ with itself shows $\left|G: G^{\prime}\right|=e^{2} n$. Thus $e=n=1$ and $G^{\prime}=G$. Hence $S=G$ proving (1.2).

To determine the structure of $N$ we need a lemma.

Lemma. If $x \in G$ and $\chi(x) \neq 0$, and $u$ and $v$ are involutions then $x=u_{1} v_{1}$ for some $u_{1}$ and $v_{1}$ conjugate to $u$ and $v$ respectively. There is one class of involutions.

Proof. Suppose not. Then if $u$ and $v$ are any involutions in $G$,

$$
0=\sum \frac{\zeta(u) \zeta(v) \overline{\zeta(x)}}{\zeta(1)}
$$

where the sum is over all irreducible characters $\zeta$ of $G$ (p. 315, [8]). Since $\chi(1)=p$ and $u$ and $v$ are involutions $\chi(u) \chi(v) \neq 0$. Moreover (1.2) implies that $\chi$ is faithful so that $\chi(u) \chi(v) \overline{\chi(x)}$ is non zero and relatively prime to $p$. Also since $\chi$ is the unique character of $G$ of degree $p, \chi$ is integer valued. This gives

$$
-\ell(\chi(u) \chi(v) \overline{\chi(x)})=p\left(\ell \sum_{\zeta \in B_{0}(p)} \frac{\zeta(u) \zeta(v) \overline{\zeta(x)}}{\zeta(1)}\right)
$$

where $\ell=l \mathrm{~cm}\left\{\zeta(1): \zeta \in B_{0}(p)\right\}$ is relatively prime to $p$. The left side is an integer prime to $p$ and the right side is an algebraic integer divisible by $p$. This contradiction proves the first sentence.

Since $G$ is a simple group, $G$ contains an element $x$ of prime order $q$ different than 2 and $p$ (Burnside's Theorem 4.3.3, [8]). If $q_{0}$ is a prime ideal in the ring of algebraic integers containing $q$ then $\chi(x) \equiv \chi(1) \equiv p\left(\bmod q_{0}\right)$. Thus $\chi(x) \neq 0$. Thus if $u$ and $v$ are involutions in $G$, they have conjugates $u_{1}$ and $v_{1}$ respectively such that $u_{1} v_{1}=x$. Hence $u_{1}$ and $v_{1}$ are conjugate in the dihedral group $\left\langle u_{1}, x\right\rangle$, and so $u$ and $v$ are conjugate.

(1.3) $N$ is dihedral of order $2 e$.

Proof. Since $B$ is a Frobenius group $B$ has $t$ non-linear characters $\varphi_{1}, \ldots, \varphi_{t}$ of degree $e$ and $e$ linear characters $\mu_{1}, \ldots, \mu_{e}$. Each $\varphi_{i}=\left(\xi_{i}\right)^{B}$ for some non-identity character $\xi_{i}$ of $P$. (See 4.5.3, [8] for example.) Now $\chi$ is fixed under field automorphisms since it is the unique character of $G$ of degree $p$. Since each $\xi_{i}$ is algebraically conjugate to $\xi_{1}$, we have

$$
a=\left(\left.\chi\right|_{P}, \xi_{1}\right)_{P}=\left(\left.\chi\right|_{P}, \xi_{i}\right)_{P}
$$

for all $i$. Moreover since $\chi$ is faithful $a \neq 0$. Thus by Frobenius reciprocity $a=\left(\left.\chi\right|_{B}, \varphi_{i}\right)_{B}$ for all $i$. Since $\chi(1)=p$ and $\varphi_{i}(1)=e, a=1$. Thus

is some linear character $\mu$ of $B$.

$$
\left.\chi\right|_{B}-\sum_{i=1}^{t} \varphi_{i}
$$


Now let $x$ be a generator of $H$.

$$
\chi(x)=\mu(x)+\sum_{i=1}^{t} \varphi_{i}(x)=\mu(x) \neq 0,
$$

since $\varphi_{i}=\xi_{i}^{\boldsymbol{B}}$ implies that $\varphi_{i}$ vanishes off of $P$. Thus the lemma implies that there is an involution $u \in G$ such that $x^{u}=x^{-1}$. Thus $\langle x, u\rangle$ is a dihedral subgroup of $N$ of order $2 e$ and so is equal to $N$.

\section{$\S 2$. Some Characters of $G$}

Recall that if $A$ is a subgroup of $G$ which is a $T I$ set then the mapping from the set of generalized characters of $N(A)$ whose support is contained in $A-\{1\}$ to generalized characters of $G, \alpha \vdash \rightarrow \alpha^{G}$, the induced character, is an isometry $(4.4 .6,[8])$.

(2.1) Suppose e $>4$. Then there are two irreducible characters, $\lambda_{1}, \lambda_{2}$ of $N$ of degree 2 which are induced from linear characters of $H . \lambda_{1}$ and $\lambda_{2}$ are real valued. There exist irreducible characters $\gamma_{1}, \gamma_{2}, \alpha$ of $G$ and integers $\varepsilon$ and $\delta$ of absolute value 1 so that

$$
1_{H}^{G}-\lambda_{i}^{G}=1-\varepsilon \gamma_{i}+\delta \alpha .
$$

$\gamma_{1}(x)=\gamma_{2}(x)$ unless $x$ is conjugate to an element of $H-\{1\}$.

Proof. The first two sentences are elementary since $N$ is a dihedral group. A proof of the last, which is well known, is included. Frobenius reciprocity gives $\left(\Gamma_{i}^{G}, 1_{G}\right)=1$ where $\Gamma_{i}=1_{H}^{N}-\lambda_{i}$. Applying the isometry to $\Gamma_{i}$ gives $\left(\Gamma_{i}^{G}, \Gamma_{j}^{G}\right)_{G}=2+\delta_{i j}$. Thus

$$
\Gamma_{i}^{G}=1+\delta \alpha+\varepsilon_{i} \gamma_{i}
$$

where $\alpha, \gamma_{1}, \gamma_{2}$ are distinct irreducible characters and $\delta, \varepsilon_{1}, \varepsilon_{2}$ are integers of absolute value 1. Moreover $\varepsilon_{1} \gamma_{1}-\varepsilon_{2} \gamma_{2}=\Gamma_{1}^{G}-\Gamma_{2}^{G}$ has degree zero while $\gamma_{i}(1)>0$. Thus $\varepsilon_{1}=\varepsilon_{2}$ proving (2.1) if we set $\varepsilon=-\varepsilon_{1}$.

We now must recall some facts about the principal block of $G, B_{0}(p)$. No assumption about $e$ is made. Brauer's paper [1] is the reference. (Alternatively see [13].)

(2.2) There are $e+t$ characters in $B_{0}(p)$. The first $e$ of them $\zeta_{1}, \ldots, \zeta_{e}$ have the property that $\zeta_{i}$ has constant value $a_{i}$ on all p-elements and $a_{i}$ is either 1 or -1 . As a result $\zeta_{i}(1) \equiv a_{i}(\bmod p)$. The remaining $t$ make up the so called exceptional class, $\theta_{1}, \ldots, \theta_{t}$. If $g \in G$ has order prime to $p$, then $\theta_{i}(g)=\theta_{j}(g)$. $\sum_{i=1}^{t} \theta_{i}$ has constant value $a$ on the p-elements, $a=1$ or -1 , and as a result $t \theta_{i}(1) \equiv \sum \theta_{i}(1) \equiv a(\bmod p)$. Moreover $a \theta_{1}+\sum_{i=1}^{e} a_{i} \zeta_{i}$ vanishes on $p$-regular elements. $B_{0}(p)$ gives a tree $T$ in the following way. 
The vertices of $T$ are the characters $\zeta_{1}, \ldots, \zeta_{e}, \theta=\sum \theta_{i}$, and two vertices are joined by an edge if and only if they have a modular constituent in common. Two vertices are joined by at most one edge. If two vertices are joined by an elge, they take different values on p-elements.

(2.3) Suppose $e>4$. Then $\alpha=\chi ; \gamma_{1}$ and $\gamma_{2}$ are real valued characters of degree $p+1$.

Proof. At least one $\gamma_{i}$ is not in the exceptional family of $B_{0}(p)$ for if they both were, then (2.1) and (2.2) would say that they are equal everywhere contrary to $\gamma_{1} \neq \gamma_{2}$. Let $x=\gamma_{1}(1)$ and $a=\alpha(1)$. Then (2.1) implies $0=1-\varepsilon x+\delta a$, and $(2.2)$ implies that $\varepsilon x \equiv \pm 1(\bmod p)$. If $\varepsilon x \equiv 1(\bmod p)$, then $p \mid a$ and so $\alpha \notin B_{0}(p)$. Thus $\alpha=\chi$. If $\varepsilon x \equiv-1(\bmod p)$ then $\delta a \equiv$ $-2(\bmod p)$. Since $e>4$ and $e \mid p-1, p \geqq 7$. Thus $\delta a \neq \pm 1$ and so $\alpha$ must belong to the exceptional family of $B_{0}(p)$. But then $t \delta a \equiv \pm 1(\bmod p)$ which gives $-2 t \equiv \pm 1(\bmod p)$. Hence $p \leqq 2 t+1$. But $e>4$ and $e t=p-1$ yield $4 t<2 t$ a contradiction.

Since $\alpha=\chi,(2.1)$ gives that

$$
\gamma_{i}=\varepsilon\left(1+\delta \chi-\Gamma_{i}^{G}\right)
$$

$\chi$ is rational valued since it is the only character of degree $p$, and (2.1) says that $\lambda_{i}$ and hence $\Gamma_{i}^{G}$ are real valued. Thus $\gamma_{i}$ is real valued. Its degree $\varepsilon(1+\delta \chi(1))=\varepsilon+\varepsilon \delta p$. Since this must be a positive number, $\varepsilon \delta=1$. Thus $\gamma_{i}(1)$ is $p-1$ or $p+1$.

Suppose $\gamma_{i}(1)=p-1$. Then $\gamma_{i}(g)=-1$ if $g$ is a $p$-element, and so $\gamma_{i \mid P}+1_{P}$ is the regular character of $P$. In the notation of the proof of (1.3) we then have $1=\left(\gamma_{i \mid P}, \xi_{j}\right)_{P}=\left(\gamma_{i \mid B}, \varphi_{j}\right)_{B}$ by Frobenius reciprocity, and so $\gamma_{i \mid B}=\sum_{j=1}^{t} \varphi_{j}$ which vanishes on $H-\{1\}$. But if $x$ is a generator of $H$ we have $\gamma_{i}(x)=-1+\chi(x)-1_{H}^{G}(x)+\lambda_{i}^{G}(x)=-3+\chi(x)+\lambda_{i}^{G}(x)$. But $\chi(x)$ is a rational root of unity from the proof of (1.3), and $\lambda_{i}^{G}(x)$ is easily computed to be a real number $<2$. Thus $0=\gamma_{i}(x)<-3+1+2=0$ a contradiction. Thus $\gamma_{i}(1)=p+1$ proving (2.3).

For the remainder of this section assume $e>4$. By a theorem of Tuan (Theorems A and B, [14]) the tree $T$ described in (2.2) can be drawn so that it is symmetric with respect to a stem (an open polygonal subgraph) and so that mapping a character to its contragredient is the automorphism of the tree which is reflection through the stem. Thus a character is real valued if and only if it is a vertex on the stem. The trivial character is one end point of the stem. Both of $\gamma_{1}$ and $\gamma_{2}$ are on the stem and so least one of them, $\gamma_{1}$ say, has more than one modular constitutent. No modular constituent of $\gamma_{1}$ is the trivial modular representation since $\gamma_{1}(g)=1$ for a $p$-element $g$ by (2.2). Thus one of the modular constituents of $\gamma_{1}$ is a faithful irreducible representation of $G$ in a field of characteristic 
$p$ of degree no larger than $(p+1) / 2$. Feit's theorem (Theorem 1, [6]) implies that $G$ is of type $L_{2}(p)$ and so (1.2) implies that $G \cong P S L_{2}(p)$.

\section{\$3. $e \leqq 4$}

It is possible to reduce the cases $e=2,3$ and 4 to high powered classification theorems, but with very little extra effort these cases can be disposed of by elementary methods.

By (2.2) there are $e+t$ characters in $B_{0}(p)$ and there is just one other, the Steinberg character $\chi$. Hence $G$ has exactly $e+t+1$ conjugacy classes. By (1.1) $t$ of these classes are classes of $p$-elements and there is one class for the identity. Writing $|G|=(1+r p) e p$, where $1+r p=|G: N(P)|$, each $p$-element $g$ is in a class of size $|G: C(g)|=[(1+r p) e p] / p=(1+r p) e$. Thus there are $(1+r p) e t=(1+r p)(p-1) p$-elements in $G$. Let $x_{1}, \ldots, x_{e}$ be the orders of the elements in each of the remaining $e$-classes and $c_{1}, \ldots, c_{e}$ the orders of the centralizers of these elements. Summing the orders of the conjugacy classes we get

or

$$
(1+r p) \text { e } p=1+(1+r p)(p-1)+(1+r p) \text { e } p\left[\frac{1}{c_{1}}+\cdots+\frac{1}{c_{e}}\right]
$$

$$
1=\frac{1+r(p-1)}{1+r p} \cdot \frac{1}{e}+\frac{1}{c_{1}}+\cdots+\frac{1}{c_{e}} .
$$

Suppose $e=2$. Then $N$ is the centralizer of an involution and has order 4. Since $G$ is simple, $G$ has a prime $q$ different from 2 and $p$ in its order. The centralizer of a $q$-element is a power of $q$, since $G$ has only 3 classes of $p$-regular elements. Thus we may write $c_{1}=q^{n}$ and $c_{2}=4$. Eq. $(*)$ becomes

$$
1=\frac{1+r(p-1)}{1+r p} \cdot \frac{1}{2}+\frac{1}{q^{n}}+\frac{1}{4} .
$$

This forces $q^{n}<4$ and $q^{n}=3$ first of all and then forces $r=1$ and $p=5$. Thus $|G|=60$ and so $G \cong P S L_{2}$ (5). (See [7] for example.)

Suppose $e=3$. Since $N$ has order 6 and is dihedral the three elements are self centralizing and conjugate to their inverses. This says that the 3-Sylow subgroups have order 3 and that there is one class of 3-elements. By the lemma of Section 2 there is one class of involutions. Thus we may write $c_{3}=x_{3}=3, x_{2}=2$ and $c_{2} \geqq 4$. Eq. (*) yields that

$$
1<\frac{1}{3}+\frac{1}{c_{1}}+\frac{1}{4}+\frac{1}{3}
$$

which gives $c_{1}<12$. By the above $c_{1} \neq 2,3,6$, or $9 . c_{2}$ cannot be 10 because then $G$ would contain elements of order 5 and 10 , and there are not enough classes for this. Neither can $c_{1}$ be 5,7 or 11 because if $c_{1}=x_{1}=q$ 
for a prime $q$, then a $q$-Sylow subgroup $Q$ has order $q$, and its normalizer induces the full automorphism group of $Q$ since there is only one class of $q$-elements. This forces $G$ to contain an element of order $q-1$ and there are not enough classes for such an element. Thus $c_{2}=2^{a}$ and $c_{1}=2^{b}, a$ and $b \geqq 2$. The only solution consistent with $*$ is $c_{1}=4$ and $c_{2}=8$. Applying (*) again yields $r=1$ and $p=7$ giving $|G|=168$. Thus $G \cong P S L_{2}$ (7). (See [7] for example.)

Finally suppose $e=4$ for purposes of obtaining a contradiction. Since $H$ is characteristic in $N$, and $N$ is dihedral of order $8, N$ is a 2-Sylow subgroup of $G$ and $H$ is self centralizing. Moreover there is just one class of elements of order 4 by Sylow's theorem. By the lemma of Section 2 there is just one class of involutions. Thus we may wirte $x_{2}=2$ and $c_{2} \geqq 8, x_{4}=c_{4}=4$ and $c_{3} \geqq c_{1} \geqq 3$ with neither $c_{1}$ nor $c_{3}$ being 4 . Eq. (*) gives

$$
1<\frac{1}{4}+\frac{2}{c_{1}}+\frac{1}{4}+\frac{1}{8}
$$

which says that $c_{1}=3$ or 5 . Hence $x_{1}=c_{1}=q$ where $q$ is 3 or 5 . Thus a $q$-Sylow subgroup has order $q$ and $c_{2}=8$.

This gives just two possibilities for $c_{3}$ and $x_{3}$. Either $c_{3}=x_{3}=q$ or $x_{3}=s$ a prime different from $p, 2$ and $q$ and $c_{3}=s^{n}$ for some $n$. If $c_{3}=q$ then Eq. $(*)$ becomes

$$
1=\frac{1}{4}\left(\frac{1+r(p-1)}{1+r p}\right)+\frac{2}{q}+\frac{1}{4}+\frac{1}{8}
$$

with $q$ either 3 or 5. $q=3$ gives no positive integer solutions to $r$ and $p$ while $q=5$ implies $r=1$ and $p=9$ a contradiction.

On the other hand if $x_{3}=s$ then the normalizer of an s-Sylow subgroup is a Frobenius group of order $\left(s^{n}-1\right) s^{n}$. (This follows from the Burnside fusion theorem and the fact that $c_{3}=s^{n}$.) Inspecting the possible Frobenius complements (see 10.3.1, [8] for example) yields that $s^{n}-1=2$ or 4 and so $c_{3}=3$ or 5 . Thus we may assume $c_{1}=3, c_{2}=8, c_{3}=5$, and $c_{4}=4$, and equation (*) has no solution with these values for the $c_{i}$. This completes the proof of the theorem.

\section{§4. The Corollary}

The corollary follows directly from the theorem. Since $G$ is a transitive permutation group of degree $p$ where $p$ is a prime, $G$ is isomorphic to a subgroup of the symmetric group on $p$ letters. Thus $p$ divides $|G|$ only once and the $p$-Sylow groups of $G$ are self centralizing. But a $p$-Sylow subgroup being self centralizing implies that every character whose degree is prime to $p$ belongs to $B_{0}(p)$ (Theorem 3, [2]). Thus the hypo- 
theses of the theorem hold for $G$ and $p$, and so $G \cong P S L_{2}(p)$. Since $G$ has a subgroup of index $p$, a theorem of Galois implies that $p=2,3,5,7$ or 11 (II, 8.28, [10]), and $p=2$ and 3 are excluded since $G$ is simple.

Ito proves the following related result (Theorem, [11]). Suppose $G$ is a transitive permutation group of prime degree $p$. (A) Suppose $(p-1) / 2=q$ is a prime. (B) Suppose exactly one character of $G$ has degree divisible by $p$. Then $p=5,7$, or 11 and $G \cong P S L_{2}(p)$.

Hypothesis $(B)$ is weaker than the corresponding hypothesis about the Steinberg character in the corollary. However hypothesis (A) is certainly stronger than the corollary's hypothesis about $H$. In fact it is not hard to prove that if $G$ is a simple transitive permutation group of degree $p=2 q+1$ with $p$ and $q$ primes and if $H$ is defined as in the corollary, then $H$ is a $T I$ set and $|N(H)|=2|H|$. Thus perhaps some strong assumption on $H$ is inevitable.

\section{References}

1. Brauer, R.: On groups whose order contains a prime number to the first power I. Amer. J. Math. 64, 401-420 (1942).

2. Brauer, R.: Some applications of the theory of blocks of characters of finite groups I. J. Algebra 1, 152-167 (1964).

3. Curtis, C. W., Reiner, I.: Representation theory of finite groups and associative algebras. New York: Interscience 1962.

4. Curtis, C.W.: The Steinberg character of a finite group with a $(B, N)$-pair. J. Algebra 4, 433-441 (1966).

5. Dagger, S.W.: On the blocks of the Chevalley groups. J. London Math. Soc. (2) 3, 21-29 (1971).

6. Feit, W.: Groups with a cyclic Sylow subgroup. Nagoya Math. J. 27, 571-584 (1966).

7. Frobenius, G.: Über Gruppen des Grades $p$ oder $p+1$. Sitzber. PreuB. Akad. Wiss., (1902), 351-369.

8. Gorenstein, D.: Finite groups. New York: Harper and Row 1968.

9. Humphreys, J.E.: Defect groups for finite groups of Lie type. Math. Z. 119, 149-152 (1971).

10. Huppert, B.: Endliche Gruppen I. Berlin-Heidelberg-New York: Springer 1967.

11. Ito, N.: A note on transitive permutation groups of degree $p=2 q+1, p$ and $q$ being prime numbers. J. Math. Kyoto. Univ. 3-1, 111-113 (1963).

12. Richen, F.: Blocks of defect 0 of a split $(B, N)$-pair. J. Algebra 21, 275-279 (1972).

13. Thompson, J.G.: Vertices and sources. J. Algebra 6, 1-6 (1967).

14. Tuan, H.F.: On groups whose orders contain a prime to the first power. Ann. of Math. 45, 110-140 (1944).

Prof. F.A. Richen

Department of Mathematics

University of Michigan

Ann Arbor, Michigan 48104

USA 